\title{
Asignación de recursos humanos por competencias en anestesiología: modelo del Hospital Regional de Alta Especialidad del Bajío
}

\author{
Rocío Mendoza-Trujillo* \\ Hospital Regional de Alta Especialidad del Bajío, León, Gto., México
}

\begin{abstract}
RESUMEN
Introducción: En la actualidad, el anestesiólogo forma parte de un equipo altamente especializado. Para ello ha adquirido conocimientos y habilidades, ya sea mediante una subespecialidad o por áreas de expertise en su práctica diaria; sin embargo, el saber «hacer» no asegura los resultados que busca la organización. Planteamiento: La asignación de recursos humanos por competencias es una metodología que permite determinar qué competencias son claves para contribuir a un desempeño superior, individual y grupalmente, además de incluir la organización en el sistema hospitalario. Cada competencia se asocia a una buena práctica, y estas resultan en evidencia medible. Argumento: En el Servicio de Anestesiología y Algología del Hospital Regional de Alta Especialidad del Bajío (HRAEB), la implementación de este modelo en los últimos cuatro años ha mejorado el proceso para asignar el personal idóneo en el sitio correcto, lo que se ha traducido en un incremento de la productividad, indicadores de morbilidad menores al estándar y diseño de capacitaciones, además de un mejor clima organizacional y disminución del ausentismo no programado. Conclusiones: Los Servicios de Anestesiología son subsistemas grandes en número y en potencial que son favorecidos mediante la asignación de recursos humanos por competencias.
\end{abstract}

Palabras clave: Anestesiología. Administración por competencias. Asignación de recursos humanos.

\begin{abstract}
Background: Currently, the anesthesiologist is part of a highly-specialized team, acquiring knowledge and abilities through a sub specialty program or during the daily practice. However, to have the expertise in patient treatment is very different from doing it according to the hospital's organizational requirements. Competence-based human-resource allocation is a method that allows to identify core abilities in order to achieve a high standard of performance, both individually and as a group, involving the whole team in the hospital system. Each competence is related with a good
\end{abstract}

\section{Correspondencia:}

${ }^{*}$ Rocío del C. Mendoza-Trujillo

Hospital Regional de Alta Especialidad del Bajío

Blvd. Milenio, 130

Col. San Carlos La Roncha

Date of reception: $27-02-2018$

C.P. 37660, León, Gto., México

Date of acceptance: 29 -05-2018

E-mail: rociomendozat@yahoo.es

DOI: 10.24875/HMCM.M18000004 
practice and results in measurable evidence. Methods and Results: During the last 4 years, implementation of this model in the Department of Anesthesiology and Algology in our hospital has improved the human-resource allocation process. By assigning the best anesthesiologist to the right place, this approach has resulted in an increase in productivity, a low morbidity rate compared to international standards, the design of improved training programs, an improved organizational atmosphere, and reduction of unscheduled employee absenteeism. Conclusion. Anesthesiology departments are large sub-systems inserted in a bigger hospital system, that will benefit from the implementation of the competence-based human-resource allocation model. (Hosp Med Clin Manag. 2018;11:79-85)

Corresponding author: Rocío del C. Mendoza-Trujillo, rociomendozat@yahoo.es

Key words: Anesthesiology. Competence management. Human resource allocation.

\section{INTRODUCCIÓN}

La anestesiología es una especialidad médica que ha progresado a la par de los requerimientos de otras especialidades. Su influencia va más allá de los quirófanos, involucrándose en procedimientos diagnósticos o terapéuticos que no ameritan el área quirúrgica, pero necesitan ansiólisis (sedación mínima) y/o analgesia para poder ser realizados con éxito. De este modo, se puede encontrar un anestesiólogo en cada piso de grandes hospitales, buscando cumplir criterios de seguridad con estándares ya establecidos y, casi simultáneamente, con la practicidad de una rápida recuperación posterior a un procedimiento anestésico.

De manera tradicional, los anestesiólogos son asignados a esta creciente demanda hospitalaria en un rol laboral rotatorio, es decir, semanal o mensualmente el anestesiólogo permanece en un área específica asignada y se mueve a la siguiente de acuerdo a ese rol, tomando en cuenta que hay una cédula profesional de por medio que avala que es pluri-potente, casi como una célula embrionaria, se espera que pueda convertirse en lo que sea de acuerdo a su localización: neurólogo, neonatólogo, pediatra, geriatra, intensivista, cardiólogo, etc.

Pero qué sucede en un hospital de alta especialidad, donde el anestesiólogo forma parte de un equipo subespecializado para cada patología, envuelta en un contexto de enfermedades cronicodegenerativas o, en el extremo, enfermedades congénitas, que ponen en jaque toda la fisiología con la que se cuenta para la planeación del evento anestésico, ¿cuál es el efecto en el profesional de este desequilibrio entre lo que se plasma en su perfil laboral y lo que él puede ofrecer de acuerdo a sus competencias?

Las subespecialidades de anestesiología son promovidas en este contexto, sin embargo, hablamos de conocimientos y habilidades obtenidas, es decir, estáticas, pero con potencial de crecimiento. Estos subespecialistas necesitarán, además de este periodo extra de capacitación, formar parte de organizaciones que detecten estas competencias, las utilicen para el desarrollo personal y se alcance mayor eficacia organizacional2,3.

Gestionar el estrés es una competencia que debe obtenerse, incluso, desde la formación en la especialidad. El ambiente laboral de alta presión del anestesiólogo per se genera niveles de estrés superiores a otras especialidades médicas. De acuerdo con Calebrese ${ }^{4}$ la incidencia del estrés laboral entre los anestesiólogos varía entre el 59 y el $96 \%$, mientras que en la población médica en general es de aproximadamente el $28 \%$. Sin duda, el paciente y aspectos medicolegales contribuyen, sin embargo, los profesionales de la especialidad también identifican aspectos del sistema de trabajo y la organización laboral como factores estresantes, desencadenantes del síndrome de burnout, con tasas superiores a otros profesionales de la salud ${ }^{4,5}$.

Entonces, nos encontramos frente al reto de organizar un grupo de profesionales en un entorno de alta presión con tareas múltiples y cambiantes mientras son ejecutadas. ¿Puede ser válido buscar funcionalidad en la asignación de recursos humanos por rol? Existe la posibilidad de restar valor a los anestesiólogos pensando así desde un mando medio, minimizando su quehacer diario a realizar tareas desconectadas del objetivo de este grupo de trabajo altamente especializado ${ }^{6}$. Lynda Gratton, consultora británica en comportamiento organizacional, advierte que, de no tomar las medidas necesarias para validar el desempeño y compromiso del personal, estos pueden desaparecer sin siquiera percatarnos de ello.

Modificar el concepto de un servicio hospitalario aislado para lograr proyectarse como un subsistema requiere 
dejar de lado una visión funcionalista por estrategias; el medio para hacerlo en anestesiología es la gestión humana y la asignación por competencias, una metodología que nos ha permitido diseñar e identificar los perfiles del personal, gestionar su capacitación y desarrollo, que sean participes de los objetivos organizacionales e incluirse, finalmente, en el plan de su sistema hospitalario con lo mejor que tiene como profesional ${ }^{7}$.

\section{PLANTEAMIENTO}

\section{Definir competencias en el área de la salud}

Las competencias son habilidades aplicadas. Este concepto ligado a la calidad tiene por objetivo lograr que las personas asignadas a determinada actividad sean las mejores para hacerlo. Es cierto que se busca reclutar a los mejores en una empresa y esto puede ser complicado partir desde una plantilla ya conformada; definir las competencias laborales claves (core) ayuda a la organización a un desempeño superior, porque admite que algo no funciona bien o puede ser mejor. Ya que no existe un modelo único para su implementación, es necesario definir un concepto válido para el anestesiólogo. Primero tomemos cuatro definiciones de competencia laboral que se acercan más al profesional de la salud:

- Capacidad real para lograr un objetivo o un resultado en un contexto dado (Cinterfor/OIT 1995).

- Aptitud de un individuo para desempeñar una misma función productiva en diferentes contextos y según los requerimientos de calidad esperados (Conocer, 1997).

- Conjunto de conocimientos, procedimientos, actitudes y capacidades que son personales y se complementan entre sí, de manera que el individuo puede actuar con eficacia frente a situaciones profesionales (Isus, 2002).

- Combinación integrada de conocimientos, habilidades y actitudes que conducen a un desempeño adecuado y oportuno en diversos contextos (OPS/OMS 2002).

En el año 2015 la Agencia de Calidad Sanitaria de Andalucía, España, involucra a los anestesiólogos en

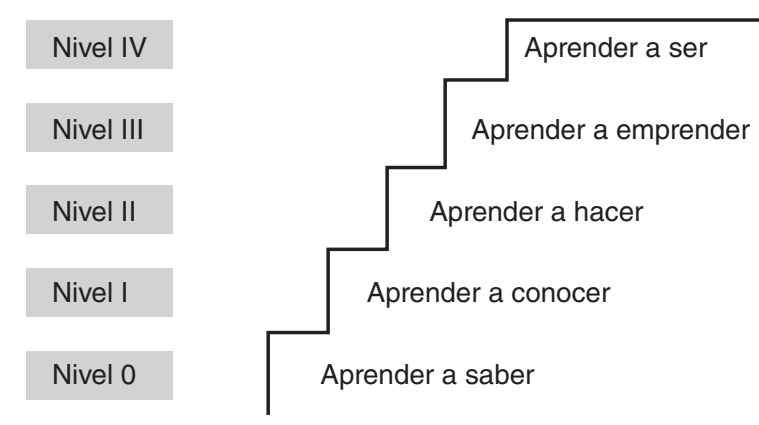

Figura 1. Niveles de competencias (adaptada de Correa BJ, $\left.2007^{9}\right)$.

un programa de certificación de competencias profesionales buscando su integración en un proceso de mejora continua ${ }^{8}$. En este texto hay una definición que integra los conceptos anteriores: la aptitud del profesional sanitario para integrar y aplicar los conocimientos, habilidades y actitudes asociados a las «buenas prácticas» de su profesión para resolver las situaciones que se le plantea. Para que esto sea posible, se reconocen cinco componentes en la evolución de la competencia, es decir, lo que el profesional «hace»: saber (conocimientos), saber hacer (habilidades), saber ser (actitudes), querer hacer (motivación) y poder hacer (aptitud profesional y medios). Estos componentes pueden esquematizarse también como una escalera que debe reconocerse tanto por el administrativo como por el operativo $^{9}$ (Fig. 1).

La asignación por competencias requiere un conocimiento profundo de la organización, reconocer en cada anestesiólogo la presencia de estas herramientas y el nivel en el que se encuentra, más aún, si está dispuesto a seguir escalando. Situarlo donde pueda dar un paso más en su desarrollo profesional es un trabajo arduo, con los retos que supone para un mando medio la diversidad laboral, por ejemplo: un Servicio de Anestesiología puede definirse productivo y funcional entre más áreas de influencia hospitalaria tenga, lo que refleja que su gente ha permitido, con base en sus capacidades, diversificar sus servicios. Las asignación por competencias es el motor de esta diversidad, de una organización heterogénea pero agrupada en labores donde se sienten cómodos y seguros en su profesión. Esta información o su análisis debe ser compartida con el profesional.

De acuerdo a las definiciones españolas, cada competencia se asocia a una buena práctica, y estas resultan 
en evidencia medible, como número de atenciones, cobertura en áreas de apoyo, uso eficiente de los recursos y la calidad de la atención. De la misma manera, debemos considerar a los integrantes del subsistema, el clima organizacional (relaciones interprofesionales) predominante, el trabajo en equipo y su participación en la enseñanza e investigación. La organización debe permitir al anestesiólogo un flujo permanente en la actualización de habilidades y conocimientos, esto le ofrecerá oportunidades de automotivación al renovarse en nuevas áreas de expertise o al reforzar una ya adquirida ${ }^{10}$.

En México, el bloque SQE (capacitación y competencias del personal), como parte del Modelo de seguridad del paciente del Consejo de Salubridad General ${ }^{11}$, solicita a la organización definir las competencias del personal necesarias para cubrir las necesidades del paciente y otorgar servicios de calidad, tomando en cuenta la misión, visión, valores de la organización, el tipo de población y los servicios que ofrece con la estructura y tecnología de que se dispone. Existen dos procesos, el reclutamiento/selección del personal y la asignación/ reasignación del personal, que ameritan el análisis de esas competencias.

Para este fin, distinguimos tres tipos de competencias: básicas, específicas y trasversales. En el reclutamiento, se toman en cuenta las competencias básicas y específicas definidas para el puesto; generalmente este análisis de cumplimiento lo realiza el área de recursos humanos con visto bueno de las jefaturas. En cuanto a la asignación del personal, sobre todo en anestesiología, es necesario reconocer si el profesional dispone de competencias transversales, aquellas que le permiten adaptarse a su contexto laboral, sea el que sea, con seguridad en su entorno y cierta autonomía. Algunos ejemplos de ellas son la comunicación efectiva, el análisis crítico y las decisiones con base en valor ${ }^{12}$.

\section{ARGUMENTO: EL MODELO DEL HRAEB}

Desde el nacimiento del Servicio de Anestesiología hace 10 años y a medida que fue integrándose la plantilla con las subespecialidades requeridas, el modelo de asignación de personal por roles no se adaptaba a la visión y estructura del hospital, y encontramos en la gestión humana la mejor estrategia para optimizar el funcionamiento del Servicio. Esto significó identificar las fortalezas y debilidades individuales para conformar equipos de trabajo reales con el resto de las especialidades. Cabe mencionar que los primeros intentos distaron mucho de una metodología clara, sin embargo, la madurez del grupo de trabajo permite actualmente una mejor concepción.

En estructura, el hospital cuenta con siete quirófanos generales y dos de cirugía ambulatoria, además de apoyar 15 servicios en auxiliares diagnósticos y terapéuticos. En capital humano, el grupo de anestesiólogos lo conforman 33 profesionales de la especialidad y cinco subespecialidades (cardioanestesiología, seis; neuroanestesiología, dos; anestesia pediátrica, tres; medicina del enfermo en estado crítico, dos, y algología, dos) que son asignados en las áreas hospitalarias mostradas en la tabla 1.

Partiendo de la infraestructura, el proceso de implementación se planeó en cinco fases en un periodo de cuatro años: 1) capacitación del mando medio en el concepto; 2) identificación de la estrategia alineada a la visión y misión del Servicio de Anestesiología; 3) identificación de las competencias básicas y específicas en la plantilla actual, y su registro en los perfiles de puesto, general y específico; 4) reclutar el personal con las competencias identificadas, y 5) evaluación de las competencias laborales definidas

Para poder identificar las competencias de los anestesiólogos, decidimos hacer el análisis desde un enfoque constructivista, esto es, las competencias laborales del anestesiólogo debían permitirle tener capacidad de improvisar con un margen de seguridad, además de cooperar y comunicarse con sus compañeros de manera efectiva. Así, fue construyéndose una matriz con 18 ítems que es susceptible de modificaciones, de acuerdo al plan de trabajo anual y/o a oportunidades detectadas por el propio personal (Fig. 2). La suma de estos puntos arroja un porcentaje que se traduce en los diferentes niveles de la escalera de competencias.

\section{Competencias laborales identificadas (Fig. 2)}

Las competencias laborales identificadas pueden diferenciarse en básicas y transversales específicas:

- Competencias básicas: certificación de la especialidad vigente, programa 0 incidencias no programadas (faltas o retardos injustificados), disponibilidad de 
Tabla 1. Servicios de apoyo de anestesiología en el HRAEB

\begin{tabular}{lll}
\hline Servicios de apoyo programado & Periodicidad & Turnos \\
\hline Cirugía ambulatoria (2) & Lunes a sábado & Matutino y vespertino \\
$\begin{array}{l}\text { Endoscopias } \\
\text { (altas, bajas, CPRE) }\end{array}$ & Lunes a viernes & Matutino y vespertino \\
Hematooncología & Lunes a viernes, domingo & Matutino y vespertino \\
Broncoscopias & Lunes a viernes & Matutino y vespertino \\
RM y TC & Lunes a viernes, domingo & Matutino y vespertino \\
Hemodinamia & Lunes a viernes & Matutino y vespertino \\
(Cardio y neurointervencionismo) & & \\
Litotricia y urodinamia & Lunes a viernes & Matutino y vespertino \\
Gabinetes & Jueves y por solicitud & Matutino \\
(Neurofisiología, ECOTE) & \\
Radioterapia & Por solicitud & \\
Atención en hospitalización & Continuo & \\
Medicina nuclear & Por solicitud & \\
(PET, SPECT) & Por solicitud & \\
Radiología intervencionista & Por solicitud en hospitalización, dos días quirúrgicos para intervencionismo en quirófano central y \\
Algología & entre semana en consultorio y admisión continua; consulta externa de lunes a viernes en diurno \\
Quirófanos centrales (7) & Continuo & \\
\hline
\end{tabular}

Fuente: Diagnóstico situacional Subdirección de Servicios Quirúrgicos HRAEB 2017. CPRE: colangiopancreatografía retrograda endoscópica; RM: resonancia magnética; TC: tomografía computarizada; ECOTE: ecocardiograma transesofágico; SPECT: tomografía computarizada por emisión monofotónica; PET: tomografía por emisión de positrones.

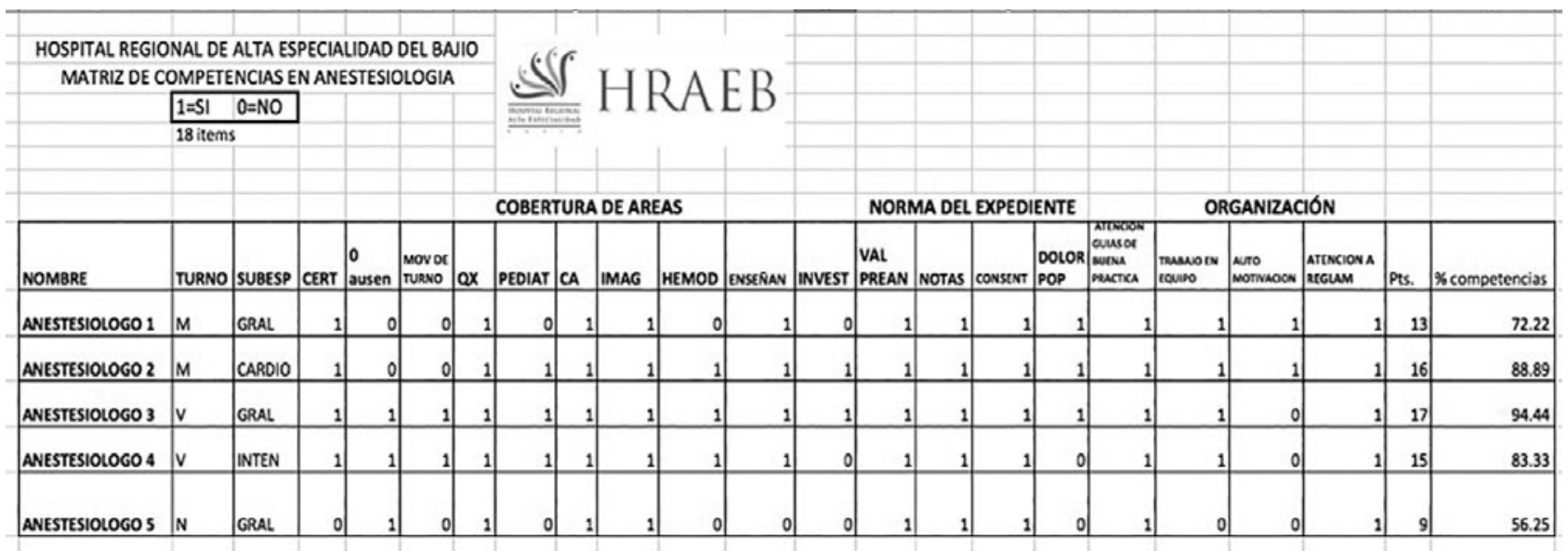

Figura 2. Matriz de competencias en anestesiología HRAEB 2017.

horario (apoyo en incidencias programadas), cobertura de áreas de apoyo (flexibilidad), aplicación de la NOM SSA 006-2011 para la práctica de la anestesiología (normatividad).

- Competencias transversales específicas: entiende cómo su trabajo se proyecta a su entorno, practica la comunicación efectiva, atiende y prioriza las guías de buena práctica en anestesia, fomenta el trabajo en equipo (interactúa y es líder en el programa de atención al dolor agudo postoperatorio con otros Servicios hospitalarios), adopta reglamentos y políticas internas en su práctica diaria (amerita poca supervisión).

\section{Procedimiento de asignación}

La asignación de recursos humanos se realiza semanalmente, este ejercicio lo realiza la jefatura tomando en cuenta en primera instancia el paciente y sus necesidades, el servicio que solicita el apoyo y el conocimiento individual del capital humano disponible que genera esta matriz. Si es indispensable la reasignación del personal, especialmente en casos clínicos complejos, el 


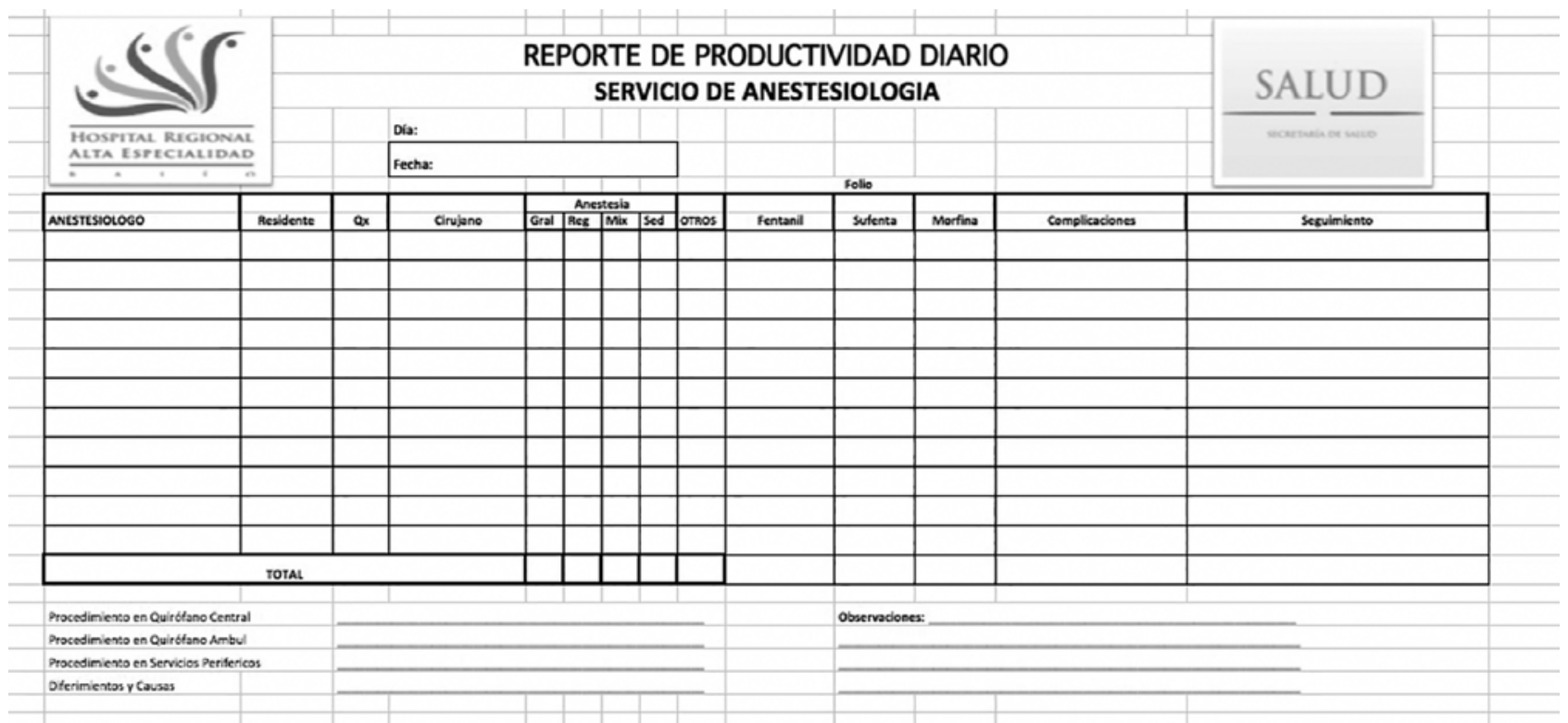

Figura 3. Reporte diario de distribución del personal HRAEB 2017.

movimiento tiene mayor éxito al conocer los diferentes perfiles del personal por medio de la matriz, es decir, visualizar opciones. Inclusive desde el planteamiento al personal operativo de los movimientos, ellos reconocen su importancia en la asignación. Estos movimientos se registran en la hoja diaria construida para medición de la productividad individual y conocimiento de necesidades específicas de los turnos (Fig. 3). La evaluación de las competencias específicas se realiza mensualmente para el área de recursos humanos y participa en un programa de reconocimiento y estímulos económicos. Sin embargo, no debe perderse de vista el objetivo inicial, identificar y potenciar las mejores habilidades y aptitudes de los anestesiólogos en pro de una atención a la salud con calidad.

\section{Evaluando el impacto}

Desde hace cuatro años, la productividad se mantiene en un crecimiento constante con un porcentaje del $11 \%$ en promedio, aunque hay áreas donde el incremento llega hasta el $45 \%$, asociado a la demanda laboral e integración de nuevos equipos de trabajo como el código ICTUS o stroke, código infarto, o incluso como resultado de un proyecto de mejora que genera mayor demanda de apoyo, como fue el ejemplo del área de imagenología donde se realizaron ajustes en las agendas de acuerdo con la plantilla de anestesiólogos que resultó en mayor número de procedimientos diagnósticos en niños que ameritaban ansiólisis. También podemos ejemplificar mejoras dentro de la propia organización del Servicio, como el fortalecimiento de la Clínica de Dolor o la Unidad de Dolor Agudo, que participaron en el crecimiento de atenciones en hospitalización y áreas de apoyo como parte del estándar COP 8 para el tratamiento del dolor en el paciente hospitalizado. Todos estos proyectos son posibles cuando el potencial humano está comprometido con los objetivos comunes (Fig. 4).

En anestesiología, las complicaciones asociadas directamente al evento anestésico es un referente de calidad. Estas complicaciones se dividen en menores (no repercuten en el pronóstico, por ejemplo, náusea y vómito), intermedias (pueden prolongar la estancia sin secuelas) y mayores (con secuelas graves). Haciendo referencia a complicaciones de rango intermedio, en 2016 este indicador fue del $0.77 \%$ y en 2017 del $0.83 \%$ (estándar 0.5 al $1.5 \%)^{13}$.

\section{CONCLUSIONES}

Los Servicios de Anestesiología son subsistemas grandes en número y en potencial; funcionan como servicio de apoyo que interactúa prácticamente con todo el resto de los subsistemas hospitalarios. El mando medio debe tener mayor claridad en la asignación del anestesiólogo, conocer dónde puede aplicar sus habilidades y conocimientos e, incluso, incrementarlos. En el HRAEB, el conocimiento obtenido al identificar las competencias 
Tendencia apoyo de anestesiología y algología HRAEB

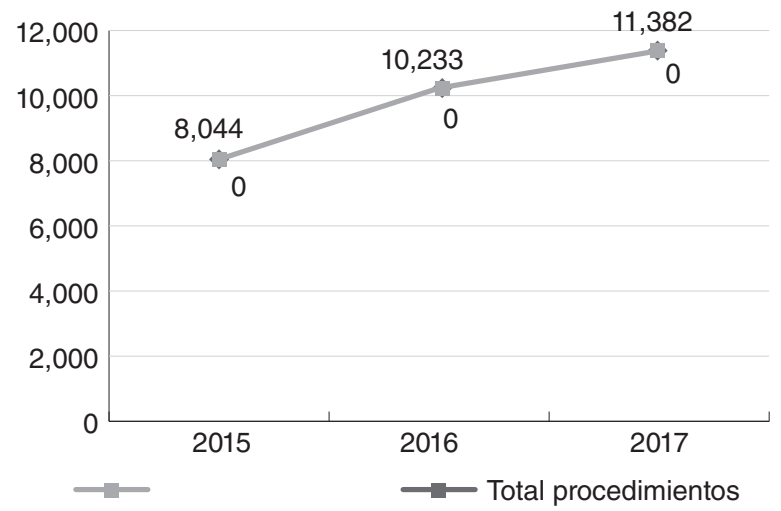

Figura 4. Tendencia en la productividad de Anestesiología y Algología 2015-2017 (fuente: Diagnóstico situacional Subdirección de Servicios Quirúrgicos HRAEB 2017).

necesarias de los anestesiólogos de acuerdo con su entorno laboral ha favorecido el desempeño grupal e individual, haciéndolo participe en los proyectos de mejora continua como parte de su bienestar laboral.

\section{BIBLIOGRAFÍA}

1. Merry AF, Cooper JB, Soyannwo O, Wilson IH, Eichhorn JH. International standards for a safe practice of anesthesia 2010. Can J Anaesth. 2010;57(11):1027-34.

2. McIndoe AK. Modern anaesthesia training: is it good enough? $\mathrm{Br} J$ Anaesth. 2012;109(1):16-20.

3. Kinzl JF, Knotzer H, Traweger C, Lederer W, Heeidegger T, Benzer A. Influence of working conditions on job satisfaction in anaesthetists. $\mathrm{Br} J$ Anaesth. 2005;94(2):211-5.

4. Calebrese G. Guía de prevención y protección de los riesgos profesionales del anestesiólogo. Anest Analg Rean. 2006;20(2):2-40. Calabrese G. Estrés laboral en el anestesiólogo. Rev Argent Anestesiol. Simposio 2012;70(1):141-8.

5. Smith AF, Glavin R, Greaves JD. Defining excellence in anaesthesia: the role of personal qualities and practice environment. $\mathrm{Br} J$ Anaesth. 2011; 106(1):38-43.

6. Alles M. Desarrollo del talento humano basado en competencias. Buenos Aires: Ediciones Granica; 2007

7. Manual de competencias profesionales. Anestesiólogo/a. España: Consejería de igualdad, salud y políticas sociales. Agencia de Calidad Sanitaria de Andalucía; 2015.

8. Correa Bautista JE. Orígenes y desarrollo conceptual de la categoría de competencia en el contexto educativo. Colombia: Editorial Universidad del Rosario; 2007.

9. Haller G, Robbins PS, Judge TA. Comportamiento organizacional. Retos y oportunidades del comportamiento organizacional. México: Pearson Educación; 2004.

10. Modelo de seguridad del paciente del Consejo de Salubridad General. Estándares para implementar el modelo en hospitales, 3 . $^{\mathrm{a}}$ edición. México: Consejo de Salubridad General; 2015.

11. Vargas Zúñiga F, Irigoin ME. Competencia laboral. Manual de conceptos, métodos y aplicaciones en el Sector Salud. Montevideo: Organización Internacional del Trabajo (Cinterfor/OIT); 2002.

12. Haller G, Laroche T, Clergue F. Morbidity in anaesthesia: Today and tomorrow. Best Pract Res Clin Anaesthesiol. 2011;25:123-32. 